\title{
Kritische Bemerkungen zur Hymenopteren-Fauna des nordwestlichen Südamerika.
}

\author{
Von \\ W. A. Schulz, Munchen.
}

Fine der letzten Arbeiten des vor Jahresfrist verstorbenen Nestors der deutschen Hymenopterologie, Dr. J. Kriechbaumer, die in dieser Zeitschrift, 1900, Seite 97-107 (mit Tafel I), erschien, behandelte die von Ihrer Königlichen Hoheit Prinzessin Therese von Bayern 1898 aus Sudamerika mitgebrachten Hymenopteren, ausser den Ameisen. Leider unterliefen dem hochbetagten, damals schon körperlich leidenden und mit Arbeiten uberbăuften Gelebrten einige Bestimmungsfehler, die ich nach Untersuchung der Belegexemplare in der Sammlung Ihrer Königlichen Hoheit Prinzessin Therese mit Höchstderen Genehmigung in folgendem richtigstelle. Die Verdienste Kriechbaumers, der jahrzehntelang fast als einziger die Schlupfwespenkunde weiterfuhrte und ihr durch seine 180 Schriften daruber endlich zu etwas allgemeinerer Anerkennung verhalf, werden durch diese meine Berichtigung nicht gescbmalert.

Der genannte Autor stellte in seiner eingangs erwahnten Abhandlung eine neue Gattung Aeanthobracon auf, die bisher nicht mebr zitiert worden ist. Ich wurde auf diese bereits vor längerer Zeit aufmerksam. als ich eine Braconide aus Ecuador erhielt, die offenbar zu ihr gehorte. Aber erst kürzlich beim zufälligen Durchblattern des Atlasses zu Lepeletiers Histoire Naturelle des Insectes Hyménoptères fiel mir die grosse Aehnlichkeit jener Gattung mit dem dort auf Tafel 44 Fig. 6 dargestellten Myosoma hirtipes Brullé auf. Die Abbildung sowohl als auch die zugehðrige Beschreibung gaben genau alle diejenigen Merkmale wieder, die Kriechbaumer als charakteristisch fur sein Genus hervorbebt, namentlich die verdickten, grob behaarten Hinterbeine und das eigentumlich gebildete erste Hinterleibssegment. Volle Gewissheit taber die Identităt von Acanthobracon mit Myosoma, von welch' letzter Gattung nach dem Dalla Torre'schen Katalog schon 
neun Arten beschrieben sind, sollte ich nun unlängst bekommen, als mir durch I. K. H. Prinzessin Therese in zuvorkommendster Weise sämtliche Typen und Originalexemplare zu der Kriechbaumerschen Arbeit zwecks Ueberprufung zur Verfugung gestellt wurden, wofur ich Ihrer Königlichen Hoheit auch an dieser Stelle meinen ehrerbietigsten Dank ausspreche. Hierbei ergab sich. dass Acanthobracon lagopus Krrchb. nicht nur generisch, sondern auch artlich hinfällt: es ist nichts weiter als das of zu dem bisher nur im $\sigma$ bekannten Myosoma hirtipes Brullé. Interessant bleibt immerhin die genaue Feststellung des Vaterlandes (mittlerer Rio Magdalena in Colombien), da von Brullé als solches nur "Südamerika" angegeben, und seither die Art in der Literatur nicht wieder erwähnt wurde.

Etwas irrefuhrend ist allenfalls die Beschreibung des zuletzt genannten Autors, wonach nur die erste Halfte des Hinterleibes rot sein soll, wäbrend dieses nach der Abbildung im Atlas in seiner ganzen Lănge, bis auf die schwarze Spitze, so gefürbt ist. Ferner steht im Text die Korperlänge zu $17 \mathrm{~mm}$ angegeben, im Widerspruch zu der Figur, wonach jene nur 7 min beträgt. Es kann keinem Zweifel unterliegen, dass in beiden Fallen einzig die Abbildung als massgebend zu gelten hat, mit der denn auch die Type Kriechbaumers gut ubereinstimmt.

Bei der Besprechung der abrigen Formen in der eingangs ange7ogenen Abhandlung halte ich mich an die dort gegebene Reibenfolge.

9) Priocnemis erythroptera Kirchb. aus Ibagué in der Zentralkordillere von Colombien steht sehr nahe Salius (Mygnimia) perpunctatus Fox (Proc. Acad. Nat. Sc. Philadelphia 1897 p. 276) von Chapada in Mattogrosso, wird aber doch wol artlich davon zu trennen sein. Als Unterschiede finde ich bei erythroptera: verhăltnismăssig grössere Lange des langeren Hinterschienensporms, andere Färbung der Fuhler und der Vorderflugelspitze. Dabei ist indes zu berucksichtigen, dass Fox nur $Q$, Kriechbaumer nur ein $\sigma$ vorlag.

Zwei nahe verwandte, aber doch von beiden vorhergenannten wieder etwas verschiedene Formen mit ebenfalls sattrotgelber Flugelfärbung besitze ich aus Archidona in Ecuador (R. Haensch leg.), die so gewissermassen Bindeglieder in der Kette der oben angedeuteten geographischen Verbreitung darstellen. Wahrscheinlich handelt es sich hier um einen ganz bestimmten Formenkreis, dessen Zerlegung in Spezies und Subspezies jedoch erst an Hand eines reicheren Materials von verschiedenen Fundorten, als ich es jetzt zur Verfugung habe, möglich sein wird. Fur P. erythroptera Krchb. speciell waren zum Zwecke solcher späteren Sichtung folgende Merkinale festzuhalten: Basis aller Flugel schwarz gefärbt, Vorderflugelspitze blass, fasst furblos, nicht 
gebrăunt, zweite Diskoidalquerader nicht wie bei den ubrigen, von mir gepruften verwandten Formen, sanft nach aussen gebogen, sondern oberhalb der Mitte scharf winklig gekniet, von dem Knie geht am typischen Exemplare im rechten Vorderflugel ein Aderstumpf nach dem Aussenrande zu aus, der im linken Flugel entgegen der Abbildung 2 auf Tafel I (dieser Zeitschr., 1900) fehlt. Die Kubitalader der Ilinterflugel entspringt vor dem Ende der Afterzelle. Der Typus zeigt ferner an den inneren Augenrădern vom Grunde des Kopfschildes an bis etwa Fublerhőhe je einen gelben Lăngstreif, und an dem zweiten Bauchsegmente sind die der ganzen Formengruppe anscheinend eigentumlichen zizenförmigen Doppelhøcker, wenn auch nicht besonders stark ausgeprăgt, so doch wol angedeutet. Wenn man nach den schon besprochenen Stucken aus Ecuador schliessen darf, wird, ebenso wie bei diesen, das noch unbekannte $ᄋ$ von $P$. erythoptera, nach Art mancher Larriden, auf dem Pygidium eine eng anliegende, glănzend rotbraune Behaarung aufweisen.

11-16) Polistes canadensis (L.). Die grossen Polistes wie die neotropischen geselligen Faltenwespen uberhaupt haben in den letzten 50 Jahren fast gar keine Bearbeitung mehr gefunden. Daher kommt es, dass unsere Kenntnis von ihnen noch sehr im argen liegt, obwohl gerade diese Insektengruppe fur die moderne Systemforschuug nach geographischen Gesichtspunkten ein dankbares Feld zu sein scheint.

Polistes annularis (L.) und canadensis (L.), zwei sehr weit verbreitete Formen, sind bisher noch immer als gesonderte Arten behandelt worden, aber schon Saussure u. a. vermuteten, dass sie spezifisch zusammenfallen. Den Beweis fur die Richtigkeit dieser Annahme habe ich nun in cincm Uebergangsstuck aus Medellin in Colombien gefunden, das in der hiesigen zoologischen Staatssammlung aufbewahrt wird. Dieses hat ganz die Tracht des canadensis, d. h. rotbraunen Kopf und pechschwarzen Thorax und Hinterleib, aber gleichzeitig ist der fur $\boldsymbol{P}$. annularis charakteristische gelbe Saum am Hinterrande des ersten Hinterleibssegments in Gestalt zweier gelber Punkte angedeutet. Somit ist der letzte $\mathrm{Zweifel}$ an der Artzusammengehðrigkeit beider bisher getrennt gefuhrter Formen geschwunden, fur welche, da canadensis die Prioritat besitzt, dieser Speziesname hinfort zu stehen hat. Nichtsdestoweniger geben sich $P$. canadensis und annularis doch als Vertreter zweier gut gekennzeichneter Formenkreise.

1) Kreis des canadensis: welche als dessen typische Form zu gelten hat, bleibt einstweilen ungewiss, da die erste Beschreibung in Linnés zehnter Ausgabe des Systema naturae (1758): „vespa 
thorace squamis duabus, abdominis ferruginei segmento primo obconico" ebenso wie dessen spätere Erläuterung im "Museum Ludovicae Ulricae" vom Jahre 1764 uber die Färbung zu düftige Angabeı euthalten, um daraus Schlusse ziehen zu lassen. Nur so vicl ist sicher, dass das typische Stuck rotbraune Flugel, Kopf und Hinterleib besass und aus Nordamerika stammte. Sicher werden sich in diesem Formenkreise Subspezies unterscheiden lassen, dazu wird es aber eines ganz besonders reichen Studienmaterials mit exakten Fundortsangaben bedurfen.

2) In dem annularis-Kreise, der ebenso wie der vorhergehende, uber Nord- und Sudamerika verbreitet ist, konnen, soweit ich zur Zeit zu ubersehen vermag, drei Hauptformen auseinander gehalten werden:

a) Flugel und Hinterleib, vom zweiten Segment an, pechschwarz; Thorax entweder ebenfalls vorherrschend schwarz mit nur wenig Rotbraun oder Gelb, alsdann auch das 1. Hinterleibssegment schwarz, mit scharf abgesetztem gelbem Ringe am Hinterrande, oder rotbraun, welche Farbe sich dann auch auf den ersten Hinterleibsring erstreckt, dessen Hinterrand gleichfalls schmal gelb gesăumt ist. Vorkommen: Nordamerika, z. B. Texas.

b) Flugel heller, dunkelbrăunlich, vor dem Aussenrande breit aufgebellt; Hinterieib und Bruststiuck vorwiegend rotbraun, Unterseite und Mittelsegment schwarz. Der gelbe Saum des im ubrigen schwärzlichen ersten Abdominalsegments erscheint viel breiter als bei der Form a) und nimmt ungefăbr dic Halfte des Segmentruckens ein. Vorn ist der Saum mehrfach ausgezackt. Vorkommen: Sudamerika, z. B. Venezuela.

c) Flugel hellgelb. Bruststuck und Hinterleib durcineg bellrotbraun, die gelbe Endbinde des Ruckens des ersten Hinterleibssegments tritt scharf hervor und erfullt die Halfte dieses, ist aber vorn in der Mitte ausgerandet. Vaterland: Antillen, z. B. Martinique, St. Kitts.

Welche von den soeben erörterten 3 Formen die typische ist, babe ich noch nicht ermitteln konnen, da ich bisher nicht im stande gewesen bin, mir Linnés "Centur. insect. rar." von 1763, mit der Originalbeschreibung, zu versehaffen, soviel ist indes sicher,. dass der Typus zu einer der beiden Formen unter a) oder b) gehören wird. Dagegen fällt Form c) vielleicht mit der "var. B* Saussures (Monog. Guêp. Soc., 1853 p. 79), sicher mit Polistes cincta Lepeletier (Hist. Nat. Ins. Hymén., I [1836] p. 522) zusammen. Diesem Namen habe ich in einer gesondert erscheinenden Abhandlung ther Antillen-Immen wieder zu seinem Recht verholfen, indem ich ihn zur Bezeichnung der Antillen-Subspezies laut c) von neuem einfuhrte. 
Von den drei Dr. Kriechbaumer vorgelegenen $Q Q$ (oder $\$ \tau)$ fällt das Exemplal No. 15 aus Carácas unter meine Form b), No. 16 ist dic Subspecies c, Polistes canadensis cinctus Lep.

18) Polistes crinitus (Felt). Mit Recht ist dieser Name von Dalla Torre wieder fur americanus Fabr. eingesetzt worden, da er älter als letzter ist. Der Einwand, dass er in einer nicht systenatischen Zwecken dicnenden Arbeit veröffentlicht wurde, fallt hin, denn Felton hat seine Vespa crinita kenntlich genug beschrieben.

Von Guadeloupe speziell scheint diese sonst weit verbreitete Art bisher noch nicht bekannt gewesen zu seill, wenigstens ist sic in Ashmeads allerdings von Fehlern strotzender Liste der aus Westindien beschricbenen Hymenopteren (Trans. Ent. Soc. London 1900 p. 299 ff.) nicht aufgefuhrt.

21) Polybia atra (Oliv.) Nur dieser Name hat nach dem Prioritätsprinzip zu gelten. Das vorliegende Stlick ist offenbar cin ․ von $15 \mathrm{~mm}$ Lange und $27 \mathrm{~mm}$ Flugelspannweite. An den Hinterecken des Hinterleibsstieles erscheint ein kleimer hellgelber Fleck angedeutet. Unklar bleibt mir, weshalb Dalla Torre in seinem Katalog fur diese Art als Vaterland nur Cayenne anfuhrt, wahrend sie doch auch vorher schon in der Literatur von anderwärts her erwähnt war.

22) ist nicht P'olybia socialis Sauss., sondern eine neue Spezies, die ich zu Ehren ihrer Entdeckerin, welche diese Widmung anzunehmen gerubte, Polybia Theresiana nenne.

$q$ an $\mathcal{\tau}$. Long. corp. $11 \mathrm{~mm}$, expans. alarum $21 \mathrm{~mm}$.

Affnis $\boldsymbol{P}$. sociali Sauss., differt clipeo acquilato, antice in medio angulato, mandibulis nigris, capite abdomineque ex parte flavidovariis, alis subhyalinis, anticis tantum ad marginem anteriorem obscuratis.

Caput transversum, desuper visum, circulare, antice nitidum, supra et postice coriaceum, opacum. Oculi mandibularum basin fere attingent. Mandibulae modice robustae, sparsim grosse punctatae, apice quatuor dentibus acutis munitae. Clipe us latior quam longior, trapezoidalis, sat grosse, sed sparsim punctatus, lateribus parallelis, antice in medio in angulum triangularem productus, superficie media elevata, in medio ipso ovatim impressa. Frons latissima, tumido-elevata, modice dense punctata, sulco mediano longitudinali, pone ipsam antennarum insertionem profunde impresso. oculi in vertice longitudine antennarum flagelli articulorum primi usque ad sextum inter se distant. Ocelli in triangulo aequilaterali dispositi, posteriores inter se quarta parte minus quam ab oculis distant. Distantia eorum ab oculis longitudinem antennarum flagelli articulorum $2^{\text {di }}+$ dimid. $3^{\text {til }}$ aequat. 
Antennae breviusculae, flagellum subclavatum, articulus hujus primus rotundatus, dinidia parte secundo brevior, tertius usque ad decimum fere aequilongi, undecimus longior.

Thorax ovalis, longior quam latior, coriaceus, opacus, fine punctatus. Prothorax brevis, antice marginatus, vix angulatus. Dorsulum convexiusculum, aeque latum ac longum, margine anteriore late rotundato. Scutellum et postscutellum sat longa, paulo elevata, plana. Ala e fere hy alinae, panlo grisescentes, anticae in margine anteriore obscuratae, stigmate nervisque piceis. Alarum anticarum cellula cubitalis secunda angusta, alta, tertia altior quam latior, in vena cubitali quam in vena radiali fere duplo longior. Pedes sat longi et robusti.

Segmentum mediale breve, oblique declive, desuper visum, subcordiforme, suleo mediano longitudinali lato. Abdominis petiolus brevis, robustus, femur III longitudine non superans, depressus, in dimidio anteriore angustus, in posteriore dilatatus, hic supra leviter longitudinaliter impressus, margine postico quam dimidium tertii segmenti marginis antici haud latiore. Abdominis reliqua pars subovata, opaca.

Corpus nigruın, supra brunnescenti-albo-, infra, in pedibus et imprimis in segmento mediali argenteo-sericeo pilosum. Coloris e b u r ne i (flavido-albi) sunt: mandibularum macula basalis, clipei maculae duae laterales, pronoti margo posticus medius, petioli apex abdominisque segmentorum ventralium tertii, quarti, quinti et sexti margines posteriores.

Hab. Ad flumen Magdalenae, reipublicae Columbianae, inter "Bodega central et Honda."

Gehört in Saussures "1I. division (Jota) ${ }^{4}$ und steht in nächster Nähe von dessen Polybia socialis, ähnelt auch $P$. frontalis Fox (Proc. Acad. Philad. 1898 p. 455), unterscheidet sich aber von erstgenannter Art durch andere Kopfschildform, weisse Körperbehaarung, hellere Flaggelfärbung und Auftreten von blassgelben Flecken am Körper, von letztgenannter durch abweichende Kopfschild- und HinterleibsstielBildung, durch andere Anordung der Nebenaugen, nicht durchweg glashelle Flugel sowie andere Körperfärbung.

Kopf flach, von oben gesehen, fast kreisrund erscheinend, Mandibeln und Kopfschild stark glänzend, Stirn und Scheitel matt, lederartig. Wangen kaum angedeutet, die Netzaugen reichen vielmehr fast bis an den Oberkiefergrund. Oberkiefer massig gross, zerstreut grob punktiert, mit vier ziemlich spitzen Endzähnen. Kopfschild breiter als lang, trapezoidisch, mit parallelen Seitenrändern, uberall ziemlich grob, aber zerstreut punktiert, Oberflăche mitten etwas erhöht, auf der Erhöhung befindet sich ein eiförmiger Lăngseindruck, KopfscbildVorderrand mitten dreieckig, in einen fast spitzen Winkel vorgezogen. 
Stirn sehr breit, nicht besonders dicht, seicht punktiert, mitten mit einer schwulstartigen Erhebung, zu beiden Seiten einer feinen, vom vorderen Nebenauge bis zwisclen die Fuhler ziehenden Iangslinie eingedruckt, dieser Langseindruck wird plotzlich etwas oberhalb der Fubleransatzstelle sehr tief und erscheint dort wie reingestochen. Der geringste Netzaugen-Abstand auf dem Scheitel beträgt die lange des 1. - 6. Fuhlergeisselgliedes. Die Nebenaugen stehen in einem gleichseitigen Dreieck, die hinteren sind von einander nur ein viertel so weit als von den Netzaugen entfernt. Ihr Abstand von den Netzaugen ist gleich der Lange des 2. thalben 3 . Fuhlergeisselgliedes. Fühler, wie zumeist bei den Arten der Gattuug Polybia, tief unten, nahe am Kopfschilde eingefugt, verhăltnismăssig kurz. Fuhlergeissel gegen das Ende hin keulenformig, Glied 1 rundlich, um ungefahr die Hälfte kürzer als 2, 3-10 ziemlich gleich lang. Endglied etwas länger als diese.

Bruststuck länglich eiförmig, lănger als breit, seine Oberfläche fein lederartig, mattglänzend, uberall fein, seicht, zerstreut punktiert. Prothorax kurz, langs der Vorderecken erstreckt sich ein feiner Rand, der aber kaum winklige Ecken bildet. Mittelrucken etwas erhaben, von gleicher Lange wie Breite, sein Vorderrand, gegen das Pronotum hin, breit abgerundet, fast abgestutat erscheinend. Hier findet sich auch mitten eine nach hinten verlaufende feine Lãngslinie Parapsidenfurchen sind in Gestalt von zwei feinen, geraden vom Schildchen aus längs der Flugelschuppen verlaufenden Langslinien angedeutet. Schildchen und Hinterschildchen ziemlich lang, ein wenig erhaben, oben fast flach. Flagel fast glashell, nur wenig grau getrubt, lediglich am Vorderrande der Vorderflugel (in der Kostal-, Medial-, Submedial-, ersten Kubital- und Radialzelle) dunkelgebräunt; Flugelmal und Adern pechschwarz. Zweite Kubitalzelle im Vorderflugel hoch und schmal, dritte hoher als breit, an der Kubitalader doppelt so breit als an der Radialader. Dritte Kubitalquerader wăhrend der letzten $\% / 3$ ihres Verlaufes, von der Radialader an gerechnet, stark nach aussen vorgezogen, aber nicht gekniet. Beine ziemlich lang und kraftig.

Mittelsegment kurz, seitlich gesehen, schief abschussig, von oben betrachtet, dreieckig bis herzformig erscheinend, mitten mit breitem Längskanal. Hinterleibsstiel gedrungen, verhăltnismassigkurz, nicht langer als Schenkel III, depress, in der vorderen Hälfte schmal, in der hinteren ziemlich plðtzlich verbrejtert, gegen das Ende oben mit einem leichten Längseindruck; Hinterrand nicht breiter als die Halfte des Vorderrandes des dritten Segments (nach Kohlscher Zahlung). Hinterleib vom letztgenannten Segment an eiformig-konisch, glanzlos. 
Schwarz, Oberseite bräunlich-weiss-, Unterseite, Beine und besonders das Mittelsegment seidenglänzend silberweiss bebaart. Elfenbeinfarben (blassgelb) sind: ein Fleck am Grunde des Oberkiefers, zwei Seitenflecke am Kopfschilde, Hinterrand des Vorderruckens in der Mitte, Endrand des Hinterleibsstieles und die Endränder der Bauchsegmente 3-6 (wiederum nach der oben erwăhnten Zălweise).

Die Type befindet sich in der Sammlung Ihrer Koniglichen Holneit Prinzessin Therese von Bayern.

$\boldsymbol{P}$. socialis ist seit ihrer erstmaligen Beschreibung durch Saussure, im Jabre 1853, (aus .Brasilien"), in der ganzen Literatur nur mehr einmal wieder, 1898, durch Fox a. a. 0 . behandelt worden, dem aus der gewaltigen brasilianischen Ausbeute Herbert H. Smiths auch nur ein, aus Rio stammendes Exemplar vorlag. In meiner eigenen Sammlung besitze ich die Art nicht, ebensowenig habe ich sie unter dem Material des hiesigen zoologischen Staatsmuseums aufaufinden vermocht. Aus allen diesen Umstănden durfte es wol den Schluss zu ziehen berechtigt sein, dass $P$. socialis Sauss. sehr selten ist.

23) Polybia pallidipes (Oliv.). Die vorliegenden beiden Stücke stellen die Aberration mit ganz verblasstem gelbbraunem Hinterleibe vor.

24) und 26) sind richtig Polybia occidentalis (Oliv.), erstes, anscheinend ein $\varnothing$, mit blassem, letztes, scheinbar $\varnothing$, mit satterem Gelb. Bei dem $\widehat{\phi}$ ist die gelbe Färbung des Mittelsegments durch eine dunkle Längslinie in 2 Hălften geteilt, was einen Uebergang zu der weiblichen "var." Saussures andeutet, uur sind die gelben Binden der letzten Hinterleibssegmente nicht schmåler als bei der typischen Form.

25) Polybia fasciata Lep. Bei dieser in den Sammlungen vielfach aus Colombien steckenden Art ist es Dalla Torre (Catal, vol. IX, 1894 p. 163) entgangen, dass Polistes fasciata Lep., Encycl. méthod., 1825 nicht hierher, sondern als Synonym zu Polybia fulvofasciata (Deg.) gehört, worauf bereits Saussure, Monog. Guêp. Soc., 1853 p. 182 Fussnote 2 aufmerksam machte.

27) Zethus haemorrhoidalis Krchb. gehort in Saussures Subgenus $Z$ ethusculus (ursprthnglich von diesem Autor als II. division bezeichnet) und ist allerdings eine durch ihre rotbraunen Beine und Hinterleib sowie durch Skulpturmerkmale. sehr ausgezeichnete, aparte Art.

28) Odynerus magdalenae Krchb. muss leider ebenfalls, und zwar als Synonym von Odynerus (Pachodynerus) nasidens Latr. fallen. Kriechbaumer hat offenbar, wis aus seiner Er- 
orterung der Verwandtschaft von magdalenae hervorgeht, nur Band I von Saussures Ftudes sur la famille des Vespides aus dem Jahre 1852 zu Rate gezogen, nicht aber auch die 23 Jahre spater erschienene Synopsis of American Wasps desselben Verfassers, in der u. a. auch gerade die Gruppe $O$. brevithorax-nasidens-simplicicornis einer grundlichen Revision unterworfen wurde. Den vermeintlichell, von Kriechbaumer zum Vergleich mit seinem magdalenae herangezogenen $O$. nasidens habe ich in der Munchener Staatssammlung eingesehen. Es ist dies ein $Q$ aus der alten Guérin-Méneville'schen Sammlung, von Cuba und gehort gar nicht zu nasidens, sondern zu der den grossen Antillen eigentumlichen Form $O$. simplicicornis Sauss. Damit entfallen auch von selbst alle Schlussfolgerungen unseres Autors, nur erscheint es angebracht, seine Beschreibung: ,capite thoraceque griseo-sericeo maculatis," die ohne Einsicht der Type leicht zu Missdeutungen Anlass geben könnte, hier in "aureo-sericeo-maculatis" richtigzustellen. Ferner sind die gelben Zeichnungen am Prothorax, Postscutellum und Metanotum keineswegs undeutlich, sondern scharf abgeboben. Das einzige Bemerkenswerte an dor Type von $O$. magdalenae scheinen mir die gelben Seitenflecke auf dem im abrigen schwarz gefärbten Racken des letzten Hinterleibssegments sowie das Vorhandensein eines gelben Flecks am Grunde des Oberkiefers zu sein. Letzten hat allerdings auch $O$. simplicicornis, welche Form uberhaupt, wie auch schon Saussure andeutete, wohl lediglich eine geographische Subspecies von nasidens Latr. darstellt, und die Fărbung des Pygidiums bleibt bei so ausserordentlich variablen Arten, wie es die Odyneri dieser Gruppe sind, ohne Belang.

Saussure hat bereits wiederholt auf die bei oberflachlicher Betrachtung tauschend grosse Aebnlichkeit der Odynerus-Formen der Gruppe brevithorax-nasidens mit gewissen Nectarina-Arten hingewiesen. Diese Aehnlichkeit erstreckt sich auf Grősse, allgemeine Körperfärbung und namentlich auf die bei den korrespondierenden Arten beider Gattungen vorhandene reiche, goldgelbe Seidenbehaarung. $O$. (Pachodynerus) nasidens speciell hat als Doppelgangerin die unter No. 29) behandelte Nectarina-Art. Die Anhanger der „Mimikry"-Theorie alten Stils sollten sich diesen "Fall" nicht entgehen lassen, nur durfte ihnen hier die Konstruktion eines "Schutzes" besondere Schwierigkeiten verursachen, denn beide Wespen, die gesellige Nectarina sowol als auch der solitäre Odynerus sind Stecher, bei denen von "Nachafiung" reden zu wollen schlechterdings absurd ware. Die Erklărung für die Erscheinang der s. g. Mimikry durfte wesentlich einfacher sein, als sie sich die Verfechter der alteren Evolutionstheorie zurecht gelegt haben: jeder, der jahrelang Insekten 


\section{W. A. Schulz. Krit. Bemerk. z. Hymenopteren-Fauna.}

verschiedener Ordnungen studiert hat, weiss, dass die Natur in ihren Zeichnungsmotiven keineswegs unbeschrănkt, unerschöpflich, vielmehr verhältnismässig arm ist. Namentlich in den an Formen reicheren Tropen, aber auch in den gemăssigten Zonen treten oft in verschiedenen, manchmal systematisch weit von einander getrennten Insekten-, ja selbst in anderen Tiergruppen, z. B. Spinnen, dic gleichen oder doch thnliche Zeichnungsmuster auf. Sekundär scheint dann allerdings in manchen Fallen die naturliche Zuchtwahl eingegriffen zu haben, um wirklich schwache, wchrlose Formen durch Anpassung an den Habitus wolbewaffneter zu schutzen.

29) Nectarina mellifica Say. Das $\$$ von Puerto Berrio am Magdalenenstrom scheint wol, schon wegen des passenden Fundorts, hierher zu gehören, genau lässt es sich aber wegen seines mangelhaften Erhaltungszustandes nicht bestimmen.

Bei dieser Gelegenheit mag darauf hingewiesen werden, dass die zuletzt angefuhrte Gattung nach dem jetzt gültigen strengen Prioritătsgesetz Nectarina, wie sie auch urspringlich von Shuckard 1840 benannt wurde, lauten muss. Die Schreibweise Nectarinia, deren sich Dalla Torre, Kriecbbaumer u. a. bedienen, wurde, wenn sie richtig wăre, mit der gleichnamigen bekannten Vogelgattung Illigers (1811) kollidiren.

Die von Kriechbaumer behandelten Bienen habe ich, soweit sie ihm von unserem grossten Kenner dieser Gruppe, Herrn H. Friese, bestimmt worden sind, nicht naher gepruft. Xylocopa tricuspidifera Krchb. (No. 46), die nicht in diese Kategorie gehort, mag vielleicht eine besondere Art sein, eine Fntscheidung dartaber wird sich aber erst treffen lassen, wenn die von Friese in Arbeit genommene Monographie der Gattung $X y$ locopa veröffentlicht ist. Bis dahin sei nur so viel bemerkt, dass das vermeintliche Hauptmerkmal, nach dem der Autor auch die obige Art bennnnte, nämlich der Dreizack unter dem Kopfschilde, wertlos ist, $d a$ es bej einer ganzen Anzahl von kleineren $X y l o c o p a-$ Arten, z. B. mordax Sm., carbonaria Sm., wie es scheint, aber immer nur im weiblichen Geschlechte, vorkommt.

Bei tricuspidifera ist der Originalbeschreibung nachzutragen, dass der Hinterleib dunkelgrün gefurbt ist, und jedes Segment vor der Mitte eine ponceaufarbene Querbinde trăgt. Cieber dic Lănge des ganzen Hinterleibes verläuft ein feiner Mittelkicl. Die Flugel sind lichtbraun, am Aussenrande dunkler, hier matt blăulichgran, auf der Scheibe matt ponceaufarben schimmernd.

No. 31) ist von Friese s. Zt. nach Ausweis der Original-Etikette als fraglicb zu Trigona argent a ta Lep. gehörig, bestimmt worden, was Dr. Kriechbaumer in seinem Verzeichnisse zu bemerken ubersehen hat. 